obstruction of the superior vena cava as its main clinical characteristic. When the fibrosis develops at the hilar level, typically in the age group 15 to 35 , a mainly unilateral mass may cause only mild respiratory disability. If both hila are affected, increasing incapacity may lead to death.

The diagnosis of hilar fibrosis is often difficult, especially when the mass is not obvious radiologically. The clinical findings vary according to whether the arteries or veins are predominantly affected. ${ }^{3}$ When the arteries bear the brunt, recurrent pulmonary and pleural infections are the rule; haemoptyses may result from widespread bronchopulmonary anastomoses. Radiographs show a shift of the mediastinum to the affected side, a small pulmonary artery, and a thickened pleura. Angiograms and studies of lung function confirm the diminished arterial blood flow. Pulmonary hypertension develops when both pulmonary arteries are involved. When the veins are mainly infiltrated, severe haemoptyses may arise from capillary congestion. Pulmonary hypertension may lead to right heart failure. Radiographs usually show a small lung with venous congestion and pleural thickening. Cardiac catheterization may show an exceptionally high pulmonary artery pressure. Additional diagnostic evidence of idiopathic hilar fibrosis may come from bronchography or bronchoscopy, when a long smooth bronchial stricture may be evident owing to compression of the associated large airways by fibrous tissue. The main differential diagnosis is from primary lung cancer, and it may be resolved only at thoracotomy. Primary pulmonary venous sclerosis is not associated with a hilar mass, nor are the larger bronchi affected.

Medical treatment has nothing effective to offer. Surgical correction of obstruction is rarely feasible and attempts to dilate bronchial strictures may lead to dangerous haemorrhage. Perhaps these unfortunate patients, few as they are, will be afforded relief when more is known of the cause of this obscure malady.

\footnotetext{
${ }^{1}$ Comings, D. E., Skubi, K. B., Van Eyes, J., and Motulsky, A. G., Annals of Internal Medicine, 1967, 66, 884.

Que, G. S., and Mandema, E., American fournal of Medicine, 1964, 36, 320.

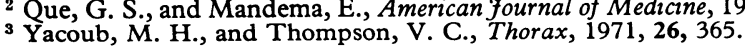

\section{Bites and Blains}

"My belief is that in very many cases the eruption is simply the result of flea bites." Sir Jonathan Hutchinson ${ }^{1}$ was not the first to suspect that most, perhaps all, cases of papular urticaria, or lichen urticatus, are caused by external parasites. The reluctance of doctors dogmatically to incriminate "insects" as the cause of papular urticaria and a variety of other pruritic eruptions is understandable. Patients are apt to resent the diagnosis, and the doctor is unable to explain how only one member of the household is affected when all seem to be equally exposed and also often cannot suggest the possible source of the alleged infestation. Most of the difficulties are resolved by a greater understanding of the immunology of reactions to insect bites and of the life histories of the species concerned.

With the exception of Hymenoptera (wasps, bees, and their allies), the substances injected by the bite of most European arthropod species do not provoke a perceptible reaction in a person not previously bitten by one of the same or related species and not allergic to one or more of the injected substances. Though some people may be repeatedly bitten without ever developing a reaction most develop allergic sensitivity which varies in type and intensity from one person to another and, with repeated exposure, in the same person. Many subjects eventually become hyposensitized or completely desensitized. The degree of sensitivity is thus one important factor influencing the clinical picture. The feeding habits of the parasite and the mode of exposure of the human host are other variables which in combination result in an extraordinarily wide range of eruptions. Occasionally pruritus is the only manifestation, but usually there are erythematous, eczematous, urticarial, papular, or even bullous lesions. Dermatitis herpetiformis may be simulated.

M. Hewitt, G. S. Walton, and $M$. Waterhouse ${ }^{2}$ have done a valuable service in drawing attention to the prevalence in man of infestations with parasites carried by domestic pets. They investigated cases of pruritic eruption referred to dermatological clinics in rural Cornwall over a period of five years. Domestic pets of the patients were thoroughly brushed on a large sheet of paper and the brushings sent for parasitological examination. The results are of considerable importance even if the percentage of patients with skin disease directly attributable to ectoparasites of animal origin is smaller in urban areas than the estimated $5 \%$ in Cornwall. The parasites most often incriminated were the flea of cats and dogs, the canine scabies mite, the mites Cheyletiella parasitovorax and C. yasguri, a variety of forage mites, and some lice. In some cases the nature and distribution of the eruption suggested its probable cause. For example, canine scabies often produced in a patient an eruption of small papules on the legs, arms, and sometimes on the face without the burrows characteristic of infestation by the human scabies mite. Cheyletiella tended to produce a polymorphic eruption of macules, papules, and vesicles followed by pustules and necrosis. More often the lesions were less distinctive. The authors note causes of missed diagnoses other than the common one of failing to consider the possibility. Many carrier animals may show no signs of infestation and may be passed as healthy by a veterinary surgeon. Moreover, the carrier may not be the patient's pet but that of friends or neighbours who are not allergically sensitive to the parasite concerned.

Other animal parasites such as rat mites and mites and lice in birds' nests on house walls can also be troublesome to man and are even more difficult to track down and eliminate. It is fair to say that the systematic exclusion of parasitic infestation is essential in every patient with a pruritic eruption of which the nature and cause are inapparent. This is the only way of effectively curing otherwise intractable and exhausting symptoms. ${ }^{1}$ Hutchinson, J., Lectures on Clinical Surgery, Vol. 1, p. 79, London,
J. and A. Churchill, 1879.
${ }^{2}$ Hewitt, M., Walton, G. S., and Waterhouse, M., British fournal of Derma-
tology, 1971, 85, 215.

\section{Treatment of Aneurysmal Subarachnoid Haemorrhage}

By percutaneous carotid and vertebral angiography we can identify during life the nature and site of a lesion causing intracranial subarachnoid haemorrhage. The commonest $(75 \%)$ and most dangerous of such lesions is an aneurysm of one of the large vessels on the cranial floor. The age and 
general and neurological condition of the patient affect the outcome of treatment, as do the site and relationship of the aneurysm and the attitude and experience of the surgeon, so that it is impossible to lay down general rules of treatment. However, some knowledge of the average mortality of the initial bleed in aneurysmal subarachnoid haemorrhage, the frequency of recurrent haemorrhage, and the effects of the subarachnoid blood on the cerebral circulation has accumulated. The possible benefits of surgery have been weighed against its risks, and various techniques of surgical treatment have been developed and evaluated.

When considering the prognosis of aneurysmal subarachnoid haemorrhage it is necessary to bear in mind that some patients die before the diagnosis is achieved, and even before admission to hospital. After admission to hospital the more seriously ill patients with spontaneous subarachnoid haemmorrhage will not be subjected to angiographic investigation, and the presence of an aneurysm will not be disclosed. Even after transfer to a neurosurgical department there is further selection, for surgical treatment will be withheld from some patients and forestalled by a fatal recurrent haemorrhage in others. This means that surgical treatment is usually offered to the less seriously ill patients in the whole group, and even among them treatment may not be attempted because of the site or multiplicity of the aneurysms.

The mortality of the initial haemorrhage has been estimated at $43 \% .^{1}$ The function of the operation is to prevent recurrent haemorrhage, and the mortality from such recurrent haemorrhage in the first 12 months after the initial one has been estimated at $35 \%$ of the survivors of the initial haemorrhage. The risk of death is greatest in the first six weeks and falls to $10 \%$ after this, being smaller still as the months pass. Clearly to minimize the risks of recurrent haemorrhage the earlier operative treatment is instituted the better. However, it is the general experience that the operative mortality in the first week or even longer may be extremely high. This applies not only to moribund or deteriorating patients but also to those who are drowsy with a minor neurological deficit. Vascular spasm and brain oedema secondary to the haemorrhage and difficult operating conditions explain this. Many surgeons therefore feel that operation must be delayed until the period of increased mortality has passed, and of course in doing so accept the risk of a further and possibly fatal recurrent bleed.

Surgical treatment will thus often be delayed for one to three weeks after initial haemorrhage. The longer the delay, the smaller the risk of death or disability from recurrent haemorrhage, and it must be ensured that the risks of surgical treatment at a late stage do not exceed those of continued conservative treatment. This point has been clearly demonstrated in a recent study. ${ }^{2}$ In a group of 92 conservatively treated patients in good general condition, with a single aneurysm which had bled on average seven weeks earlier, nine died of recurrent haemorrhage during the subsequent $3 \frac{1}{2}$ years. Of 86 patients of similar type treated surgically after a similar average period, one died in hospital and four in the next four years. It seems clear then that reparative processes occur in the aneurysmal wall in many cases, and the view that surgical treatment is required even when the aneurysm is detected many months after the haemorrhage can no longer be held.

The technique of surgical management has improved. The value of the simpler procedures of cervical carotid ligature for internal carotid aneurysms and anterior cerebral clipping for anterior communicating aneurysms has been demonstrated in the prevention of recurrent haemorrhage and by postoperative angiography. ${ }^{3} 4$ Many series of intracranial aneurysms successfully dealt with by direct attack are now on record. Occlusion of the neck of the sac or obliteration of the sac with clips is the method of choice, but investment of the aneurysm with muscle or other material has its advocates. The mortality of these procedures has reached acceptably low figures in many series. Improved results depend on many factors. The selection of the patient is one of these. Others are controlled respiration during anaesthesia, hypothermia, and hypotension at the critical period of dissection of the sac. In an occasional case the assistance of the thoracic surgeon with temporary occlusion of the venous return to the right atrium and of the aortic arch has been valuable. Without doubt the increasing use of the operating microscope in neurosurgery will facilitate the dissection necessary to occlude the neck of the aneurysmal sac. Thus progress in the surgical management of these dangerous lesions has been made and will continue. 1 Pakarinen, S., Acta Neurologica Scandinavica, 1967, 43, Supplement 29.
2 Troupp, H., and Bjorkesten, G., af., fournal of Neurosurgery, 1971, 35, 20.
3 Tindall, G., and Odom, G., in Progress of Neurological Surgery, 3. Basel,
Karger, 1969.
4 Durity, F., and Logue, V., fournal of Neurosurgery, 1971, 35, 16.

\section{Fibrin and Cancer}

Anticoagulants and fibrinolysins have been shown to reduce the growth and spread of malignant tumours in experimental animals. ${ }^{1-3}$ The induction of lipaemia, which favours the formation of fibrin, and the inhibition of fibrinolysis with epsilon-aminocaproic acid have been shown to encourage tumour growth. ${ }^{4}$ These effects may be independent of the coagulation mechanism, for in no case is the action of the experimental agent confined to that system alone, but the first hypothesis must be that fibrin formation is involved in neoplastic growth. What relevance have these studies to cancer in man?

In different experiments tumours have not been affected equally, and at least one tumour showed increased growth in heparinized animals. ${ }^{6}$ E. E. Cliffton and his associates ${ }^{1}$ have reported the benefits of a single injection of heparin. B. Fisher and E. R. Fisher ${ }^{2}$ needed to prolong the administration of heparin for up to seven days and to use much larger doses. All workers agree that the main changes are seen when the anticoagulant or fibrinolysin is given before inoculating the animal with the tumour. In some experiments the primary tumour decreases in size, but more commonly the number and size of metastases has been reduced.

R. A. Q. O'Meara7 has suggested that a fibrin latticework which he has detected around many tumours is a prerequisite for vascularization and growth of the tumour. Many tumour cells have been shown to contain coagulant factors $^{78}$ and clot-stabilizing factors 9 allied to the plasma factor XIII of the coagulation mechanism. However, such materials are constituents of normal tissue. Some tumour cells also produce fibrinolysins, ${ }^{10}$ which might be expected to have the opposite effect.

Tumour cells which enter the blood can sometimes be shown to become surrounded by fibrin and platelets. ${ }^{11}$ It has been suggested that microthrombi form and enmesh 